\title{
Review
}

\section{Searching for a more sensitive earthworm species to be used in pesticide homologation tests - A meta-analysis}

\author{
C. Pelosi ${ }^{\mathrm{a}, *}, \mathrm{~S}$. Joimel ${ }^{\mathrm{b}, \mathrm{c}}, \mathrm{D}$. Makowski ${ }^{\mathrm{d}, \mathrm{e}}$ \\ a INRA, UR251 PESSAC, F-78026 Versailles cedex, France \\ ${ }^{\mathrm{b}}$ Université de Lorraine, Laboratoire Sols et Environnement, UMR 1120 BP 172, F-54505 Vandoeuvre-lès-Nancy cedex, France \\ ' INRA, Laboratoire Sols et Environnement, UMR 1120 BP 172, F-54505 Vandoeuvre-lès-Nancy cedex, France \\ d INRA, UMR 211 Agronomie, F-78000 Thiverval Grignon, France \\ e AgroParisTech, UMR 211 Agronomie, F-78000 Thiverval Grignon, France
}

\section{H I G H L I G H T S}

Eisenia fetida is less sensitive to pesticides than species found in cultivated fields.

- Sensitivity of Lumbricus rubellus is variable from one study to another.

- Aporrectodea caliginosa and Lumbricus terrestris are the most sensitive species to pesticides.

- A. caliginosa is proposed as model for ecotoxicological and homologation tests.

\section{A R T I C L E I N F O}

Article history:

Received 5 March 2012

Received in revised form 24 July 2012

Accepted 8 September 2012

Available online 22 October 2012

\section{Keywords:}

Earthworm sensitivity

Pesticides

Homologation test

Ecotoxicological studies

LC50

Mortality

\begin{abstract}
A B S T R A C T
Pesticide risk assessments include experiments designed to measure the effect of pesticides on earthworms using the Eisenia fetida fetida or Eisenia fetida andrei species. There is no clear consensus in the literature on the sensitivity of different earthworm species to pesticides. We performed a meta-analysis on the sensitivity of several earthworm species to pesticides to determine the most sensitive species, and to discuss their suitability for European homologation tests. A dataset including median lethal dose (LC50) values reported in 44 experimental treatments was constructed and then analyzed in order to compare the sensitivity levels of $E$. fetida with that of other earthworm species. Results showed that LC50 values reported for Lumbricus terrestris and Aporrectodea caliginosa were on average significantly lower than for E. fetida. Considering the relatively high LC50 values reported for E. fetida and the absence of this species from zones where pesticides are usually applied, the relevance of using E. fetida for pesticide homologation tests is questionable and we advise risk assessors to use A. caliginosa as model species. A new protocol based on this species could be proposed for European homologation tests but its implementation will require the definition of a new standard and take time. In the meantime, the results obtained with E. fetida should be interpreted with caution taking into account the low sensitivity of this species. Our study illustrates the value of the meta-analysis approach for comparing the sensitivity of different earthworm species to pesticides. It would be useful to extend the dataset presented in this paper in order to analyze the sensitivity of other aquatic or terrestrial organism groups used for pesticide homologation or ecotoxicology tests.
\end{abstract}

(c) 2012 Elsevier Ltd. All rights reserved.

\section{Contents}

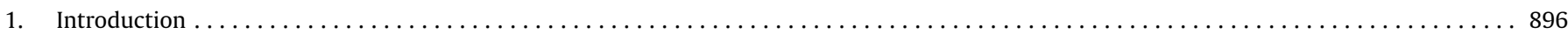

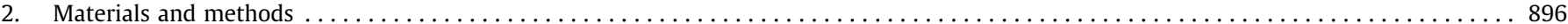

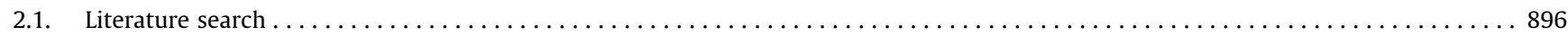

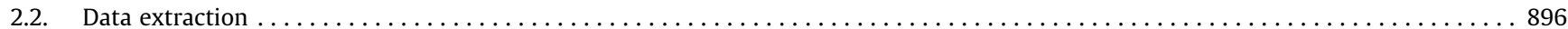

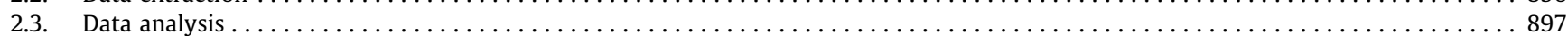

\footnotetext{
* Corresponding author. Address: UR251 PESSAC INRA, Bâtiment 6, RD 10, 78026 Versailles cedex, France. Tel.: +33 130833607 ; fax: +3313083 3259 .

E-mail address: celine.pelosi@versailles.inra.fr (C. Pelosi).
} 


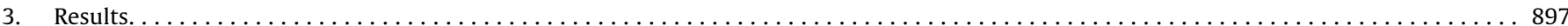

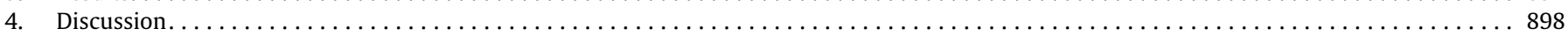

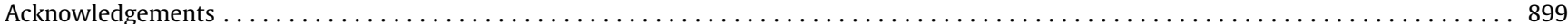

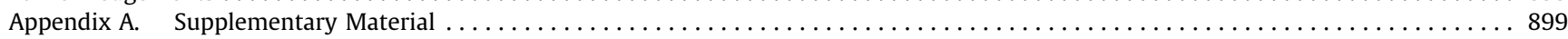

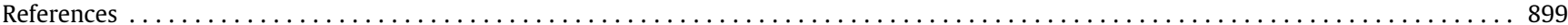

\section{Introduction}

Intensive agricultural production has led to a deterioration in soil quality (Vitousek et al., 1997). Chemical inputs, in particular pesticides, have been applied in large quantities in western European agricultural areas, and have affected soil biodiversity (Mäder et al., 2002; Bengtsson et al., 2005). In order to limit the environmental impact of pesticides, national and European food safety agencies carry out risk assessments for pesticides in order to provide scientific support to stakeholders prior to pesticide marketing authorization. Such risk assessments include experiments designed to measure the effect of pesticides on different aquatic and terrestrial organisms, such as earthworms.

Earthworms represent a large proportion of soil organism biomass and provide important agro-ecological functions necessary for the functioning of agroecosystems. Earthworms are "one of the key organisms in environmental toxicology" (Spurgeon et al., 2003). Two earthworm species Eisenia fetida fetida and Eisenia fetida andrei have been in European pesticide marketing authorization experiments since the early 1980s. In these experiments, adult individuals are exposed to active substances or commercial formulations of pesticides at different concentrations for fourteen days on filter paper or in an artificial OECD soil (ISO 11268-1, 1993; ISO 11268-2, 1998; OECD 207, 1984). OECD soil is a homogeneous mixture of $10 \%$ sphagnum peat, $20 \%$ kaolin clay, and $70 \%$ sand. The $\mathrm{pH}$ is adjusted to $6.0 \pm 0.5$ by the addition of calcium carbonate (OECD 207, 1984). Measurements of earthworm mortality are used to estimate LC50 values, i.e. the lethal concentrations for $50 \%$ of exposed individuals. Effects on reproduction are also sometimes studied in these experiments, but results are rarely made publically available (PPDB, 2011; ANSES, Agritox, 2012).

In addition to the marketing authorization tests, both subspecies of Eisenia fetida are often used in ecotoxicological studies dealing with the effects of pesticides on earthworms (Ma and Bodt, 1993; Yasmin and D'Souza, 2007). According to Yasmin and D'Souza (2007), Eisenia fetida "is especially appropriate for the toxicity tests because it can be easily bred on a variety of organic wastes with short generation times". However, there are at least two reasons to question the relevance of using this species as a reference for toxicity. Firstly, E. fetida is not found in mineral soils (Lowe and Butt, 2007) and is thus very uncommon in the cultivated fields where pesticides are applied. Other species such as Aporrectodea caliginosa or Lumbricus terrestris are present in cultivated fields and are thus exposed to pesticides. L. terrestris is more commonly found in no-tilled plots since it is sensitive to plowing (Chan, 2001; Pelosi et al., 2009). Both are ecologically important in terrestrial ecosystems of many temperate regions (Bouché, 1992; Bauer and Römbke, 1997). These species are not frequently used in ecotoxicological studies and the main reason seems to be that they are less easily bred than E. fetida (Cortet et al., 1999).

Secondly, some authors found that E. fetida is comparatively less sensitive to contaminants than other earthworm species (Roberts and Dorough, 1985; Kula, 1995; Fitzgerald et al., 1996). Stenersen (1979) reported that E. fetida could tolerate pesticide concentrations up to one hundred times higher than the lethal concentration for A. caliginosa. Consequently, the use of E. fetida as a reference species for ecotoxicological test may lead to an underestimation of earthworm mortality due to pesticides.
The lower sensitivity of E. fetida was not confirmed by Callahan et al. (1994) who found that four species E. fetida, Allolobophora tuberculata, Eudrilus eugeniae and Perionyx excavatus all had similar sensitivity to 62 different chemicals. These authors concluded that E. fetida is a representative model for these species. Pizl (1988) highlighted a strong correlation between LC50 values of E. fetida and those of Lumbricus rubellus, which is also often used in ecotoxicological tests (Spurgeon et al., 2003; Lowe and Butt, 2007). Haque and Ebing (1983) and Heimbach (1985) showed that E. fetida is of comparable sensitivity to pesticides as $L$. terrestris. Since no clear consensus can be found in the literature on the sensitivity of the different earthworm species to pesticides, it is useful to synthesize and analyze the available experimental data in order to identify the most sensitive species.

Hence, the aim of this paper was to make a meta-analysis of the sensitivity of several earthworm species to pesticides and metabolites in order to determine which species is the most sensitive and to discuss which would be most suitable for use in European homologation tests.

\section{Materials and methods}

\subsection{Literature search}

A systematic literature review was conducted to find publications dealing with earthworms exposed to pesticides. The literature search was carried out on the basis of keywords in ISI Web of Knowledge, using the "All Databases" option, with the following formula: "(earthworm* or lumbric*, aporrectod* or eisen* or dendrobaen* or allolobophor* octalas*) and (pesticide* or herbicid* or fungicid* or mollusc* or nematicid* or insecticid*)' in Topics.

From a corpus of more than 1800 references, a first selection was made using titles and abstracts. The full texts were examined when information was considered consistent for meta-analysis. To complete the search, starting from the previously selected references, authors that had written articles on the subject as well as books and journals of interest were identified and their articles were examined.

We only considered publications which provided data on E. fet$i d a$ and another species in the same study in order to compare the sensitivity of the species in the same conditions i.e. the same earthworm development stage, active substance, type of substrate, pesticide addition, applied organic matter, and experiment duration. In order to be as representative as possible of natural conditions, we only included data from natural or artificial soil tests, excluding results from filter paper or immersion tests. Papers dealing with tropical earthworm species were also excluded. It was decided to focus on lethal effects because there were not enough studies on biomass or on chronic effect of cocoon production that suited the constraints we set. Finally, we selected a corpus of 15 publications, i.e. 11 papers and 4 studies in a book chapter (Appendix A).

\subsection{Data extraction}

Data from the fifteen selected publications were entered into a relational database including several variables: author(s), year of the study, species, development stage i.e. juvenile or mature, active 
substance name, type of substrate e.g. natural soil or OECD, pesticide addition i.e. mixed or sprayed onto soil surface, applied organic matter, experiment duration, estimated LC50 value, and 95\% confidence interval of the LC50. Data were collected from the text, tables and digitized figures of the articles. The species A. tubercula$t a$, studied in one paper, was classified as A. caliginosa (Blakemore, 2006).

\subsection{Data analysis}

We defined a treatment as a unique combination of "author(s), year, development stage, active substance, type of substrate, pesticide addition, applied organic matter, and duration". We identified a total of 44 treatments, including results for several earthworm species (Appendix A). Each of the 44 treatments included LC50 values for E. fetida (E. fetida fetida or E. fetida andrei) and for one or several other species. The lower the LC50, the higher is the sensitivity of the earthworms to pesticides.

For each treatment and each earthworm species $s$, we calculated response ratios $R$ of the LC50 of the species $s\left(\mathrm{LC}^{2} \mathrm{~S}_{\mathrm{s}}\right)$ to the LC50 obtained in the same treatment for $E$. fetida $\left(\mathrm{LC}^{2} 0_{\mathrm{f}}\right)$ $\left(R=\mathrm{LC}_{50} / \mathrm{LC} 0_{\mathrm{f}}\right)$. The total number of calculated ratios was $78 . \mathrm{A}$ ratio of 1 indicates that the species $s$ has the same sensitivity to pesticide as E. fetida.

Next, we calculated $\log$ response ratios $(\log (R))$ to normalize the data for analysis and then calculated 95\% confidence intervals (CIs) for the response ratio using the method presented by Hedges et al. (1999). A log ratio lesser than zero indicates that the species $s$ was more sensitive to pesticide than E. fetida. The difference be- tween sensitivity of the two species was significant if the 95\% CIs of the log ratios did not contain zero.

The computation of the CIs of the log ratio was based on the standard deviations of the estimated individual LC50 values reported in the publications. When a LC50 standard deviation was missing, it was deduced from the bounds of LC50 confidence interval reported in the paper (we used the largest of the two bounds in case of dissymmetry). When both standard deviation and confidence interval of LC50 were missing (nine publications of 15), the missing standard deviation was set equal to the largest standard deviation reported in the selected publications; this approach allowed us to minimize the risk of underestimating the level of uncertainty associated with our calculations.

Finally, we computed the unweighted mean of the log ratios and the mean effect size (weighted average of the log ratios). The $95 \%$ confidence interval of the mean effect size was computed using the method of Hedges et al. (1999). We performed all statistical analyses using $R$ version 2.14.0 (R Development Core Team, 2011).

\section{Results}

From the 78 calculated log ratios (Fig. 1a), 11 concerned $L$. terrestris (Fig. 1b), 18 concerned A. caliginosa (Fig. 1c), 20 concerned L. rubellus (Fig. 1d), 6 concerned Allolobophora chlorotica and 1, 2 or 3 concerned Dendrobaena octaedra, Octalasion lacteum, Aporrectodea nocturna, Eisenia veneta, Aporrectodea rosea, and Aporrectodea longa.

Fig. 1 showed a high variability of the log ratios of LC50; this ratio ranged from -4.33 to 2.67 . Most of the log ratios were lesser (a) All species

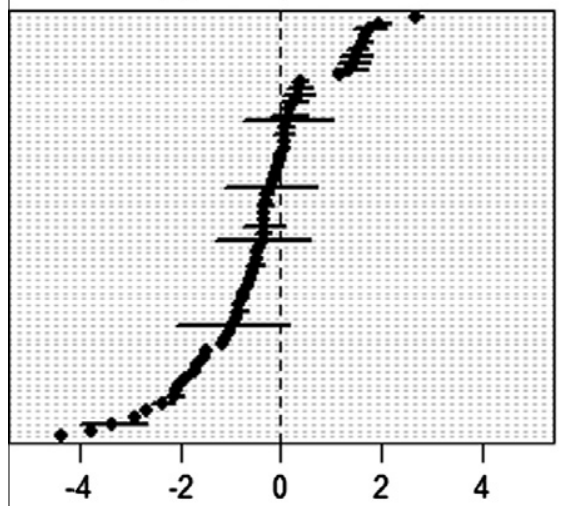

(c) A. caliginosa

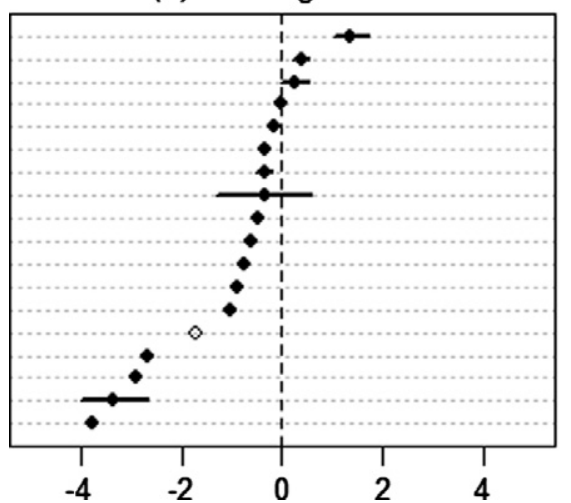

(b) L. terrestris

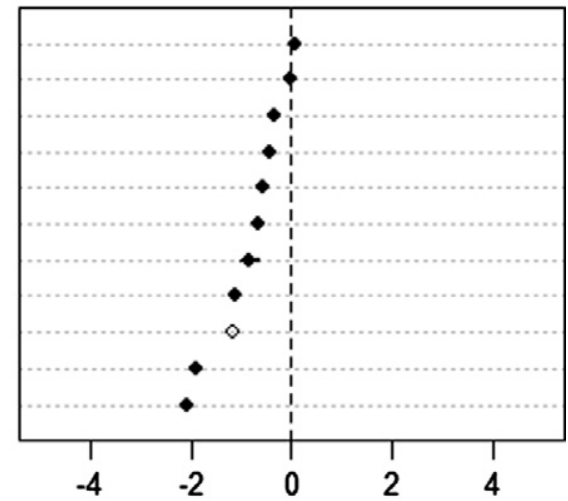

(d) L. rubellus

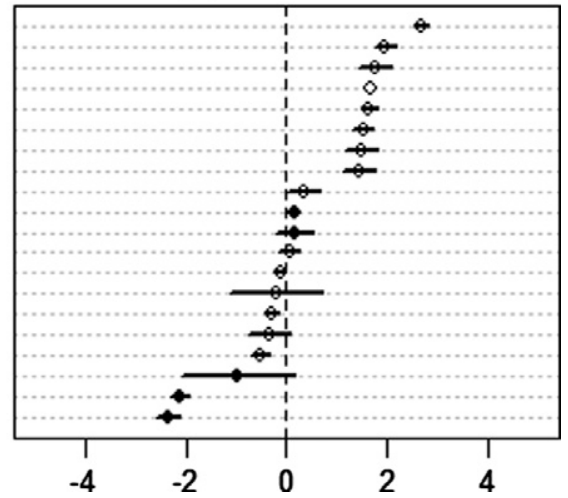

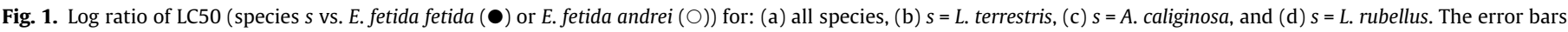
correspond to the $95 \%$ confidence intervals derived from the standard deviations reported in 6 publications (missing in the 9 other publications). 
Table 1

Mean, minimum and maximum values of log ratio, mean effect size, and confidence interval (95\%) of mean effect size calculated from the 44 experimental treatments.

\begin{tabular}{lllll}
\hline Species & $\begin{array}{l}\text { Mean } \\
\log (R)\end{array}$ & $\begin{array}{l}\operatorname{Min} ; \operatorname{Max} \\
\log (R)\end{array}$ & $\begin{array}{l}\text { Mean effect } \\
\text { size }\end{array}$ & CI (95\%) \\
\hline All & -0.72 & $-4.33 ; 2.67$ & -0.70 & $-1.05 ;-0.34$ \\
L. terrestris & -0.90 & $-2.09 ; 0.05$ & -0.90 & $-1.49 ;-0.31$ \\
A. caliginosa & -1.24 & $-4.17 ; 1.35$ & -1.23 & $-2.00 ;-0.46$ \\
L. rubellus & 0.40 & $-2.34 ; 2.67$ & 0.40 & $-0.28 ; 1.08$ \\
\hline
\end{tabular}

than zero, indicating that E. fetida is generally less sensitive to pesticides than the other earthworm species. This result is confirmed by negative values of the unweighted mean of the $78 \mathrm{log}$ ratios and of the global mean effect size equal to -0.72 and -0.70 respectively (Table 1).

Although the $95 \%$ confidence intervals of the individual log ratios frequently overlapped the zero threshold (Fig. 2a), the confidence interval of the global mean effect size value (computed over all species) ranged from -1.05 to -0.34 (Table 1 ) indicating that E. fetida was on average significantly less sensitive to pesticides than other species.

Almost all the log ratios for $L$. terrestris and $A$. caliginosa were less than zero (Fig. 1b and c, Fig. 2b and c). Although the 95\% confidence intervals of the individual log ratios overlapped the zero threshold in some cases (Fig. 2b and c), the mean log ratios, mean effect sizes and their confidence intervals confirm that $L$. terrestris and $A$. caliginosa, two species widespread in European agricultural fields, were significantly more sensitive to pesticides and metabolites than E. fetida species (Table 1). The values of mean effect size obtained for $L$. terrestris and A. caliginosa showed that the LC50 of these species was in average $41 \%(100 * \exp (-0.9))$ and $29 \%$ $(100 * \exp (-1.23))$ of the LC50 of E. fetida.

Log ratios obtained for $L$. rubellus (a species commonly used in ecotoxicological studies) were much higher than those obtained for $L$. terrestris and A. caliginosa; their values were highly variable between treatments, ranging from -2.34 to 2.67 , and the mean effect size was 0.4 (Table 1, Figs. 1d and 2d). The number of treatments was too small for the other species, i.e. A. chlorotica, $D$. octaedra, O. lacteum, A. nocturna, E. veneta, A. rosea, and A. longa, to justify a separate analysis.

\section{Discussion}

The corpus of studies selected for this meta-analysis allowed us to compare the sensitivity to pesticides and metabolites of several earthworm species. By using fifteen publications and 44 different experimental treatments, we demonstrated that $E$. fetida species are among the least sensitive earthworm species. These findings raise questions on the relevance of $E$. fetida for assessing the toxicity of some pesticides in homologation tests. E. fetida are easily bred and have been recommended as model species for almost 30 years (OECD 207, 1984). Yet, considering its fairly high LC50 value compared to other species and its absence from zones where pesticides are applied, the use of E. fetida for pesticide homologation tests is questionable.

L. rubellus, an epigeic species normally living in litter layers, is also frequently used in ecotoxicological tests (Spurgeon et al., 2003; Lowe and Butt, 2007). It is common in grassland fields but

\section{(a) All species}

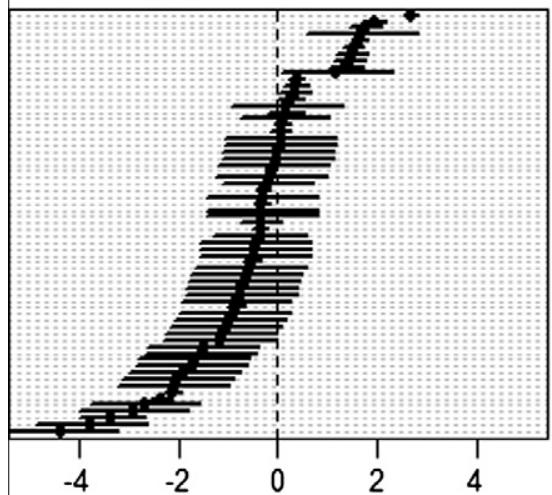

(c) A. caliginosa

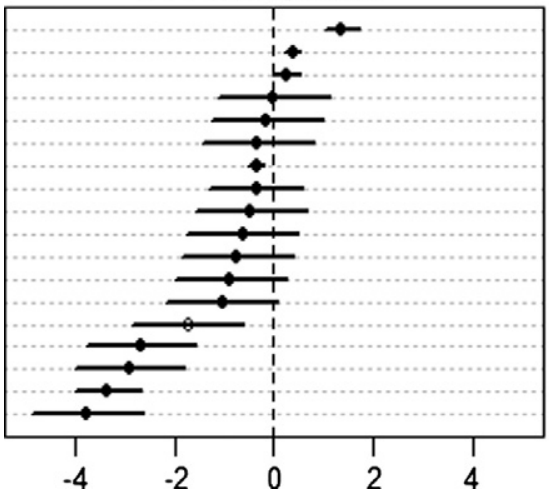

(b) L. terrestris

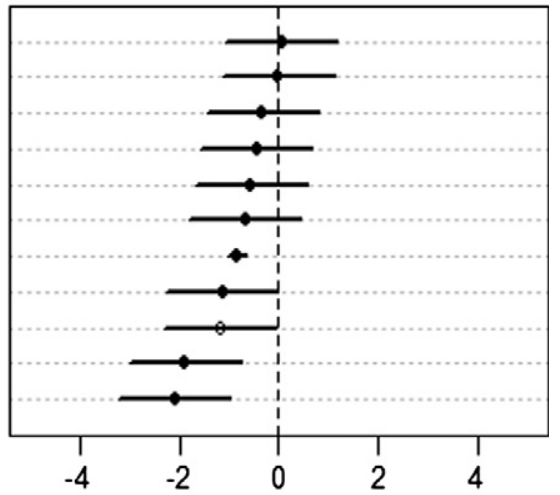

(d) L. rubellus

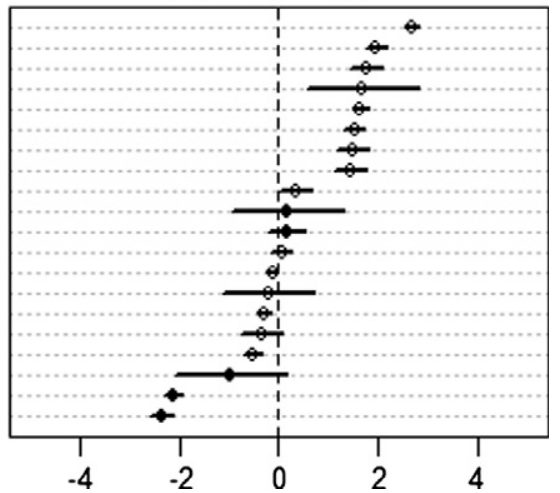

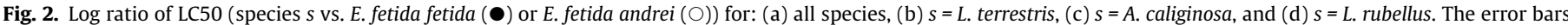

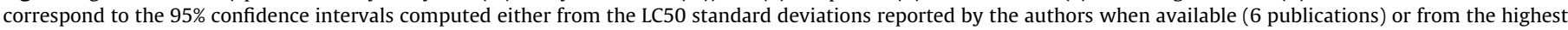
LC50 standard deviation (9 publications). 
more rarely found in conventional cultivated fields due to the lack of surface litter. In addition, our results show that the sensitivity of L. rubellus to pesticides was highly variable and, on average, lower than that of E. fetida. The sensitivity of a species may vary according to a range of factors including the active substance and soil substrate (Van Gestel and Ma, 1990, 1993; Ma and Bodt, 1993), temperature, development stage, age, pre-exposure and origin of individuals (Lowe and Butt, 2007; Zhou et al., 2008) and these variables are often poorly described in published studies.

According to the results of our meta-analysis, A. caliginosa and $L$. terrestris appeared more sensitive to pesticides than E. fetida. A. caliginosa and $L$. terrestris are obligatory soil-dwelling species widespread in European cultivated fields, L. terrestris being mainly present in unplowed systems (Chan, 2001; Pelosi et al., 2009). Moreover, $L$. terrestris is a relatively large anecic earthworm, living in deep burrows. According to guidelines proposed by Lowe and Butt (2005), sustainable culture of $L$. terrestris requires a soil deeper than $10 \mathrm{~cm}$, more than $20 \mathrm{~g}$ of dried and rewetted cattle or horse dung adult ${ }^{-1}$ month $^{-1}$ and a stocking density of 3 adults $1^{-1}$. For A. caliginosa, these criteria are about $3 \mathrm{~cm}$ for soil depth, $10 \mathrm{~g}$ adult $^{-1}$ month $^{-1}$ for food amount and 6 adults $\mathrm{l}^{-1}$ for stocking density. Moreover, A. caliginosa matures more quickly than $L$. terrestris and exhibits higher rates of fecundity (Lofs-Holmin, 1980; EriksenHamel and Whalen, 2007). Therefore, A. caliginosa seems easier to rear and more appropriate as a model species. The use of a laboratory-reared parthenogenetic soil-dwelling species such as A. caliginosa may provide a solution to the inherent variability in experimental populations that makes comparison between studies difficult. It is however important to note that A. caliginosa is a species complex (Fernandez et al., 2012) which may have a plastic phenotype. Individuals can vary greatly in size and behavior (Bouché, 1972) and this may influence the interpretation of tests undertaken using this species. The limited number of data collected on other species, i.e. A. chlorotica, D. octaedra, O. lacteum, A. nocturna, E. veneta, A. rosea, and A. longa, prevented us from drawing conclusions about their sensitivity.

A limitation of the dataset used in our meta-analysis is that standard deviations of the LC50 value were frequently missing. We decided to use the maximum of the reported standard deviation as a proxy for the missing values. However, even under such a constraint, we found significant differences between species. We strongly encourage scientists to systematically present standard deviations and/or confidence intervals of LC50 in their future papers as recommended by Philibert et al. (2012).

The observed differences of sensitivity of earthworm species to pesticides are difficult to explain (Neuhauser et al., 1986; Kula, 1995). Ma and Bodt (1993) tested and rejected the assumption that body size, i.e. surface/volume ratio, determines the level of exposure. Fitzgerald et al. (1996) explained that physiological differences between species e.g. their ability to regulate contaminant uptake or to bind xenobiotics or their metabolites in non-extractable forms, may contribute to the observed differences of sensitivity. One of the most convincing explanations is the more rapid metabolism and excretion of chemical pollutants by E. fetida compared with other species (Gilman and Vardanis, 1974).

Our study illustrates the value of a meta-analysis approach for comparing the sensitivity of different earthworm species to pesticides. It would be useful to extend the dataset used in our manuscript (Appendix A) in order to analyze the sensitivity of other groups of aquatic or terrestrial organism used for pesticide homologation or ecotoxicological tests. Daam et al. (2011) found different results when comparing E. fetida to Acari, Chilopoda, Coleoptera, Collembola, Diplopoda, Enchytraeidae, Isopoda, and Nematodes. Frampton et al. (2006) found that E. fetida was less sensitive than arthropods of Folsomia candida species for a broad range of pesticides including biocide, fungicide, herbicide, and insecticide.
Experimental data are thus available for building a larger dataset including LC50 values estimated for several species belonging to different taxa. The analysis of such a dataset would allow a limited pool of species to be identified with high sensitivity for ecotoxicological tests.

Our results show that the definition of a new test procedure based on A. caliginosa is required. However, the adoption of a new procedure by pesticide homologation agencies is likely to take time for statutory reasons. A standard protocol for culturing this species must be defined as a preliminary step. In the meantime, a simple short-term solution could be adopted; data obtained with $E$. fetida could be interpreted taking into account the fact that the LC50 of $A$. caliginosa is, in average, equal to $29 \%$ of the LC50 of $E$. fetida.

\section{Acknowledgements}

We would like to thank Folkert van Oort and Alan Scaife for thorough editorial advice in English.

\section{Appendix A. Supplementary Material}

Supplementary data associated with this article can be found, in the online version, at http://dx.doi.org/10.1016/j.chemosphere. 2012.09.034.

\section{References}

ANSES, Agritox, 2012. <http://www.dive.afssa.fr/agritox/php/fiches.php>.

Bauer, C., Rombke, J., 1997. Factors influencing the toxicity of two pesticides on three lumbricid species in laboratory tests. Soil Biol. Biochem. 29, 705-708.

Bengtsson, J., Ahnstrom, J., Weibull, A.C., 2005. The effects of organic agriculture on biodiversity and abundance. A meta-analysis. J. Appl. Ecol. 42, 261-269.

Blakemore, R.J., 2006. An updated list of valid, invalid and synonymous names of Criodriloidea and Lumbricoidea (Annelida: Oligochaeta: Criodrilidae, Sparganophilidae, Ailoscolecidae, Hormogastridae, Lumbricidae, Lutodrilidae). In: Kaneko, N., Ito, M.T. (Eds.), A series of Searchable Texts on Earthworm Biodiversity Ecology and Systematics from Various Regions of the World, second ed. COE Soil Ecology Research Group, Yokohama National University, Japan. pp..

Bouché, M.B., 1972. Lombriciens de France. Ecologie et Systématique. INRA Ann. Zool. Ecol. Anim. Publication, France.

Bouché, M.B., 1992. Earthworm species and ecotoxicological studies. In: GreigSmith, P.W., Becker, H., Edwards, P.J., Heimbach, F. (Eds.), Ecotoxicology of Earthworms. Intercept Press, Andover, pp. 20-35.

Callahan, C.A., Shirazi, M.A., Neuhauser, E.F., 1994. Comparative toxicity of chemicals to earthworms. Environ. Toxicol. Chem. 13, 291-298.

Chan, K.Y., 2001. An overview of some tillage impacts on earthworm population abundance and diversity - implications for functioning in soils. Soil Tillage Res. 57, 179-191.

Cortet, J., Gomot-De Vauflery, A., Poinsot-Balaguer, N., Gomot, L., Texier, C., Cluzeau, D., 1999. The use of invertebrate soil fauna in monitoring pollutant effects. Eur. J. Soil Biol. 35, 115-134.

Daam, M.A., Leitao, S., Cerejeira, M.J., Sousa, J.P., 2011. Comparing the sensitivity of soil invertebrates to pesticides with that of Eisenia fetida. Chemosphere 85, 1040-1047.

Eriksen-Hamel, N.S., Whalen, J.K., 2007. Competitive interactions affect the growth of Aporrectodea caliginosa and Lumbricus terrestris (Oligochaeta: Lumbricidae) in single- and mixed-species laboratory cultures. Eur. J. Soil Biol. 43, 142-150.

Fernandez, R., Almodovar, A., Novo, M., Simancas, B., Diaz Cosin, D.J., 2012. Adding complexity to the complex: new insights into the phylogeny, diversification and origin of parthenogenesis in the Aporrectodea caliginosa species complex (Oligochaeta, Lumbricidae). Mol. Phylogenet. Evol. 64, 368-379.

Fitzgerald, D.G., Warner, K.A., Lanno, R.P., Dixon, D.G., 1996. Assessing the effects of modifying factors on pentachlorophenol toxicity to earthworms: applications of body residues. Environ. Toxicol. Chem. 15, 2299-2304.

Frampton, G.K., Jänsch, S., Scott-Fordsmand, J.J., Römbke, J., Van den Brink, P.J., 2006 Effects of pesticides on soil invertebrates in laboratory studies: a review and analysis using species sensitivity distributions. Environ. Toxicol. Chem. 25, 2480-2489.

Gilman, A.P., Vardanis, A., 1974. Carbofuran. Comparative toxicity and metabolism in the worms Lumbricus terrestris L. and Eisenia foetida S. J. Agr. Food Chem. 22, 625-628.

Haque, A., Ebing, W., 1983. Toxicity determination of pesticides to earthworms in the soil substrate. Z. Pflanzenk. Pflanzens J. Plant Dis. Prot. 90, 395-408.

Hedges, L.V., Gurevitch, J., Curtis, P.S., 1999. The meta-analysis of response ratios in experimental ecology. Ecology 80, 1150-1156. 
Heimbach, F., 1985. Comparison of laboratory methods, using Eisenia foetida and Lumbricus terrestris, for the assessment of the hazard of chemicals to earthworms. Z. Pflanzenk. Pflanzens. J. Plant Dis. Prot. 92, 186-193.

ISO (International Organisation for Standardization), 1993. Effects of pollutants on earthworms (Eisenia fetida). Part 1: Determination of acute toxicity using artificial soil substrate - No. 11268-1. Geneva.

ISO (International Organisation for Standardization), 1998. Effects of pollutants on earthworms (Eisenia fetida). Part 2: Determination of effects on reproduction No. 11268-2. Geneva.

Kula, H., 1995. Comparison of laboratory and field testing for the assessment of pesticide side effects on earthworms. Acta Zoolog Fennica 196, 338-341.

Lofs-Holmin, A., 1980. Measuring growth of earthworms as a method of testing sublethal toxicity of pesticides. Swed. J. Agr. Res. 10, 25-33.

Lowe, C.N., Butt, K.R., 2005. Culture techniques for soil dwelling earthworms: a review. Pedobiol 49, 401-413.

Lowe, C.N., Butt, K.R., 2007. Earthworm culture, maintenance and species selection in chronic ecotoxicological studies: a critical review. Eur. J. Soil Biol. 43, S281S288.

Ma, W.C., Bodt, J., 1993. Differences in toxicity of the insecticide chlorpyrifos to 6 species of earthworms (Oligochaeta, Lumbricidae) in standardized soil tests. Bull. Environ. Contam. Toxicol. 50, 864-870.

Mäder, P., Fliessbach, A., Dubois, D., Gunst, L., Fried, P., Niggli, U., 2002. Soil fertility and biodiversity in organic farming. Science 296, 1694-1697.

Neuhauser, E.F., Durkin, P.R., Malecki, M.R., Anatra, M., 1986. Comparative toxicity of 10 organic-chemicals to 4 earthworm species. Comp. Biochem. Physiol. CPharmacol. Toxicol. Endocrinol. 83, 197-200.

OECD, 1984. Guideline for the testing of chemicals. No. 207. Earthworm, acute toxicity tests. Paris, France.

Pelosi, C., Bertrand, M., Roger-Estrade, J., 2009. Earthworm community in conventional, organic and no-tilled with living mulch cropping systems. Agron. Sustain. Dev. 29, 287-295.
Philibert, A., Loyce, C., Makowski, D., 2012. Assessment of the quality of metaanalysis in agronomy. Agric. Ecosyst. Environ. 148, 72-82.

Pizl, V., 1988. Interactions between earthworms and herbicides.1. Toxicity of some herbicides to earthworms in laboratory tests. Pedobiologia 32, 227-232.

PPDB, 2011. <http://sitem.herts.ac.uk/aeru/footprint/fr/index.htm>.

R Development Core Team, 2011. R: A Language and Environment for Statistical Computing, Version 2.12.2. R Foundation for Statistical Computing, Vienna Austria.

Roberts, B.L., Dorough, H.W., 1985. Hazards of chemicals to earthworms. Environ. Toxicol. Chem. 4, 307-323.

Spurgeon, D.J., Weeks, J.M., Van Gestel, C.A.M., 2003. A summary of eleven years progress in earthworm ecotoxicology. In: The 7th International Symposium on Earthworm Ecology, Cardiff, Wales, 2002. Pedobiol. vol. 47, pp. 588-606.

Stenersen, J., 1979. Action of pesticides on earthworms.1. Toxicity of cholinesteraseinhibiting insecticides to earthworms as evaluated by laboratory tests. Pestic Sci. 10, 66-74.

Van Gestel, C.A.M., Ma, W.C., 1990. An approach to Quantitative Structure-ActivityRelationships (Qsars) in earthworm toxicity studies. Chemosphere 21, 10231033.

Van Gestel, C.A.M., Ma, W.C., 1993. Development of QSAR's in soil ecotoxicology: earthworm toxicity and soil sorption of chlorophenols, chlorobenzenes and chloroanilines. Water, Air Soil Pollut. 69, 265-276.

Vitousek, P.M., Mooney, H.A., Lubchenco, J., Melillo, J.M., 1997. Human domination of Earth's ecosystems. Science 277, 494-499.

Yasmin, S., D'Souza, D., 2007. Effect of pesticides on the reproductive output of Eisenia fetida. Bull. Environ. Contam. Toxicol. 79, 529-532.

Zhou, S.P., Duan, C.Q., Wang, X.H., Michelle, W.H.G., Yu, Z.F., Fu, H., 2008. Assessing cypermethrin-contaminated soil with three different earthworm test methods. J. Environ. Sci. 20, 1381-1385. 